\section{Pericardial effusion associated with asbestos exposure}

Sylvie Trogrlic, Pierre Alain Gevenois, Marc Schroeven, Paul De Vuyst

\begin{abstract}
The case history is presented of a 60 year old man who developed a pericardial effusion. Chest radiography showed pleural thickening and calcification. Pericardiotomy was performed and revealed nonspecific inflammatory lesions. Occupational exposure to asbestos and exclusion of other causes led to the diagnosis of benign asbestos pericardial effusion.

(Thorax 1997;52:1097-1098)
\end{abstract}

Keywords: asbestos, pericardial effusion.

Exposure to asbestos is associated with both benign and malignant pleural lesions including effusion, diffuse thickening, plaques, and mesothelioma. ${ }^{12}$ Pericardial lesions including parietal pericardial plaques, ${ }^{346}$ chronic constrictive pericarditis, ${ }^{356}$ and mesothelioma ${ }^{3}$ have also been reported in relation to asbestos exposure. We report the case history of a patient who presented with acute blood stained pericardial effusion as a manifestation of asbestos related disease.

\section{Case report}

A 60 year old man was admitted because of precordial pain and shortness of breath. He had had pulmonary tuberculosis when he was 19. He had been intensively exposed to asbestos for one year in 1963 when he handled sacks containing asbestos in an insulation company. Approximately 10 years after this exposure he presented with several episodes of bilateral pleural effusion associated with shortness of breath and thoracic pain. The fluid was a bloody exudate without malignant cells. At that time bronchoalveolar lavage showed 28 asbestos bodies $/ \mathrm{ml}$ and the diagnosis of benign asbestos pleural effusion was made. The patient was recognised by the Occupational Disease Fund of Belgium and eventually developed bilateral diffuse pleural thickening.

At the present admission the only clinical sign was a pericardial rub. Laboratory blood tests revealed an increased sedimentation rate $(52 \mathrm{~mm} / \mathrm{h})$ and an increased level of $\mathrm{C}$ reactive protein $(7.8 \mathrm{mg} / 100 \mathrm{ml})$. Cardiac enzymes were within the normal range. Blood culture and a tuberculin skin test were negative. Electrocardiography showed non-specific changes during repolarisation. The AP chest radiograph showed bilateral diffuse pleural thickening associated with blunting of the costodia-

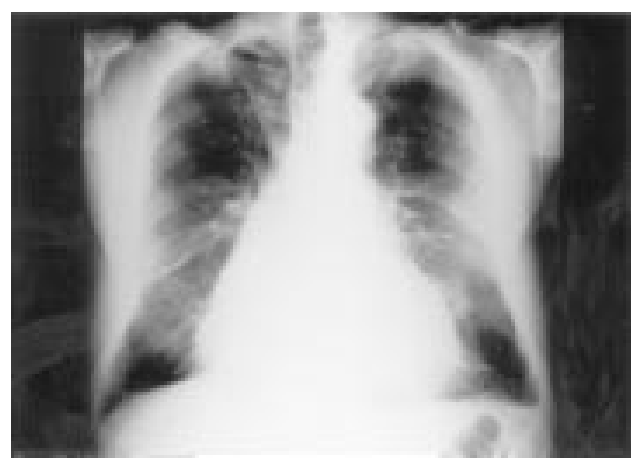

Figure 1 AP chest radiograph showing bilateral diffuse pleural thickening and blunting of costodiaphragmatic sulci.

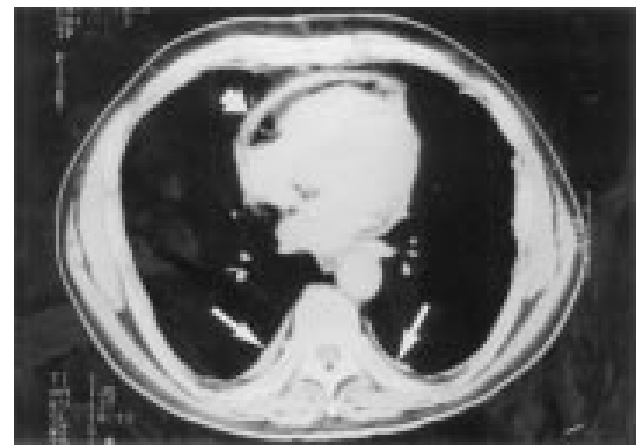

Figure 2 Chest computed tomographic scan showing pericardial thickening (short arrow) and bilateral pleural thickening (long arrows).

phragmatic sulci and an enlarged cardiac shadow (fig 1). Moreover, a computed tomographic scan of the chest showed a rounded atelectasis in the right lower lobe and pericardial thickening (fig 2). Pericardial effusion was detected by transthoracic echocardiography and was confirmed by a puncture collecting $500 \mathrm{ml}$ of a bloody fluid. Pericardial biopsy specimens taken during pericardectomy showed a non-specific inflammatory reaction. There was no recurrence of the pericardial effusion during a follow up period of two years and no signs to suggest development of constrictive pericarditis on echocardiography.

\section{Discussion}

Acute pericardial effusion related to asbestos exposure has rarely been reported. As in benign asbestos pleural effusion, the diagnosis is based on a history of asbestos exposure and by exclusion of other causes, including mesothelioma. Constrictive pericarditis has been described after exposure to asbestos and also occurs rarely, since only six cases have been reported in the English literature. ${ }^{4-7}$ Constrictive pericarditis could in some cases explain breathlessness that is disproportionately severe for the extent of asbestosis and lung function impairment. ${ }^{4}$ Magnetic resonance imaging appears superior to high resolution computed tomographic scanning in the diagnosis of this condition. ${ }^{4}$ 
The symptoms of our patient were similar during episodes of pleural effusion and when he presented with the pericardial effusion. Moreover, the pericardial and pleural inflammatory fluids had the same bloody appearance. Laboratory blood tests revealed, as is often the case in benign asbestos pleurisy, an increased sedimentation rate. ${ }^{7}$ The delay between the exposure and the pericardial effusion was approximately 30 years. In a previous case reported by Davies et $a l^{4}$ the delay was 23 years. In benign pleural effusion a delay of 10-20 years usually occurs, but delays longer than 20 years have been reported. ${ }^{17}$

It has been shown that the mesothelium lining the body cavities such as the pleura, pericardium, peritoneum, and tunica vaginalis testis have an identical disease pattern in response to common causative agents. ${ }^{8}$ How asbestos fibres can reach these cavities and, in particular, the pericardium is not well known. There are several pathways through which the fibres may migrate towards the pleura - directly through the pleural space, through the bloodstream, or via the lymphatic ducts. ${ }^{8-10}$ Direct migration of fibres from the alveoli into the pleural cavity through the visceral pleura followed by their reabsorption in the parietal pleura by particular structures that can take up particles and cells from the serosal cavity could lead to their deposition and retention in the parietal pleura. ${ }^{10}$ These structures are Wang's stomas on the parietal pleura, which allow communication between the pleural space and the subpleural lymphatics, and milky spots which are submesothelial aggregates of lymphoid cells around stomas. ${ }^{8}$ Fibres could reach the pericardium through lymphatic connections between the pleura and the peri- cardium. ${ }^{8}$ This additional step from the pleura to the pericardium could explain the longer interval between exposure and the appearance of a pericardial effusion and its low frequency compared with pleural effusions. Takada and colleagues $^{9}$ showed that injection of carbon particles into the pericardial cavity of rabbits induced a reabsorption in the submesothelial layer and, in particular, in the macrophages of milky spots. Migration of fibres through the bloodstream is also conceivable: Kanazawa et $a l^{8}$ have shown that fibres concentrated selectively in pleural milky spots after intravenous injection of asbestos.

In conclusion, exposure to asbestos should be included in the possible causes of acute pericardial effusion as well as of constrictive pericarditis.

1 Epler GR, MacLoud TC, Gaensler EA. Prevalence and incidence of benign asbestos pleural effusion in a working population. $¥ A M A$ 1982;247:617.

2 Hillerdal G. The pathogenesis of pleural plaques and pulmonary asbestosis: possibilities and impossibilities. Eur $\mathcal{f}$ Respir Dis 1980;61:129-38.

3 Davies D, Andrews MJ, Jones JSP. Asbestos induced pericardial effusion and constrictive pericarditis. Thorax 1991; cardial effusion $429-32$.

4 Al Jarad N, Underwood SR, Rudd RM. Asbestos-related pericardial thickening detected by magnetic resonance maging. Respir Med 1993;87:309-12

5 Fischbein L, Namade M, Sachs RN, Robineau M, Lanfranchi J. Chronic constitute pericarditis associated with asbestosis. Chest 1988;94:646-7.

6 Pope AR, Sokdowski JW, Spirw I. Constrictive pericarditis. Chest 1989;95:1172.

7 Hillerdal G, Özesmi M. Benign asbestos pleural effusion 73 exudates in 60 patients. Eur 7 Respir Dis 1987;71:113.

8 Kanazawa K. Exchanges through the pleura. Cells and particles. In: Chrétien J, Bignon J, Hirsch A, eds. The pleura in health and disease. New York: Marcel Dekker, 1980: 195-231.

9 Takada K, Otsuki Y, Magari S. Lymphatics and prelymphatics of the rabbit pericardium and pericardium with special emphasis on particulate absorption and milky spotspecial emphasis on particulate absorption and
like structures. Lymphology 1991;24:116-24.

10 Boutin C, Dumortier P, Rey F, Viallat JR, De Vuyst P. Black spots concentrate oncogenic asbestos fibers in the parietal pleura. Thoracoscopic and mineralogic study. Am f Respi Crit Care Med 1996;153:444-9. 